\title{
COMPARISON OF LAG-SCREW VERSUS MINIPLATE FOR OSTEOSYNTHESIS OF ANTERIOR MANDIBULAR FRACTURE: A RANDOMIZED CONTROL STUDY
}

\author{
Mohmmad A. Shuman*
}

\begin{abstract}
Objective: The aim of this study was to evaluate the outcome of mandibular fractures fixation in order to compare lag screw technique with standard miniplates.

Methods: This prospective randomized comparative study was conducted on 20 patients with mandibular anterior fractures treated with open reduction and internal fixation. The patients were then randomly allocated into two groups. Group 1: two lag screws were used for fracture osteosynthesis in 10 patients. Group 2: two miniplates were used for fracture osteosynthesis in 10 patients. The surgery was done by the same surgical team who did not involve within the research work. The time for hardware fixation was recorded intraoperatively. Patients were assessed clinically and radiographically for fracture stability, malocclusion, masticatory efficiency, mouth opening, paresthesia, pain, edema, infection, wound dehiscence, malunion/ununion and hardware loosening. A repeated measure ANOVA were used to evaluate the different between lag screw and miniplates.
\end{abstract}

Results: Hardware fixation time showed a highly significant $\left(\mathrm{p}<0.001^{*}\right)$ difference between group-1 Lag screw with an average of $15.80 \pm 0.80$ minutes and group- 2 miniplates with an average of $20.01 \pm 0.823$ minutes. The difference in interfragmentary distance between lag screw and miniplates treatments were nonsignificant $(\mathrm{p}>0.05)$. regarding occlusion, fracture stability, mastication biting efficiency and postoperative complications a non-significant $(\mathrm{p}<0.05)$ difference between both groups as revealed.

Conclusions: According to the result of this prospective study it is concluded that, the internal fixation of anterior mandibular fractures with lag screws osteosynthesis is simple, successful, achieving rapid fixation with minimal complications.

KEY WORDS: Mandibular fracture, osteosynthesis, miniplates, lag screw.

* Assistant Professor of Oral \& Maxillofacial Surgery, Faculty of Dental Medicine, Al-Azhar University, Assiut. Egypt. 


\section{INTRODUCTION}

Maxillofacial bone fractures cases have been one of the most frequent surgical cases in which oral and maxillofacial surgeons encountered overall the time. ${ }^{(1,2)}$ Among these cases, mandibular fractures are the most common with prevalence rates reported by epidemiological studies between 60 and $81 \%$ (mean 71.2). ${ }^{(3)}$ Among mandibular fractures the percentage of anterior mandibular fractures incidence $(27 \%){ }^{(4)}$ According to many literatures the etiologic factors leading to anterior mandibular fracture includes, road traffic accidents (RTA), falls, sports injuries, assaults and interpersonal aggression. ${ }^{(4,5)}$ In general the main goal of fracture management is regaining the pre-fracture anatomic shape aiming to restore the normal function and esthetic parameters as early as possible with the lowest complications. To achieve this goal in the management of anterior mandibular fractures the most recent osteosynthesis procedures, based on the theory firstly described by Champy and the AO/AOSIF have been used. These procedures have been replaced the traditional methods including, maxillomandibular fixation, splints, suspension, and external fixation which affected the quality of life by mouth closing for 6 weeks or more and wearing extraoral hard ware appliances with many recorded complications. Osteosynthesis procedures can eliminate most of these complications and improving the patient's quality of life. ${ }^{(6)}$ The original principal of Champy recommended using of 2 miniplates for internal fixation of anterior mandibular fractures is the standard routine method for management of these fractures until now. ${ }^{(7)}$ However the thick cortices, curvature shape and absence of anatomical hazards of the anterior mandible uniquely makes lag screw application appear to be simple, safe and reasonable in internal fixation of anterior mandibular fractures. ${ }^{(8-10)}$ Hence, the purpose of the present study was to compare the clinical and radiographic outcomes of osteosynthesis of mandibular anterior fracture by using of two different techniques for internal open reduction and fixation, titanium miniplates versus cortical lag screws.

\section{PATIENTS AND METHOD}

This is a randomized prospective study conducted on patients who presented in the Department of Oral and Maxillofacial Surgery, Al-Azhar university, with clinical and radiological diagnosis of anterior mandibular fractures. The anterior mandibular fracture is recognized as the fracture at the area between the two mental foramina. Twenty patients were selected for this study. The inclusion criteria were no significant medical history, noncomminuted discrete isolated fractures of the anterior mandible without evidence of infection. Exclusion criteria were comminuted fractures, multiple mandibular fractures, panfacial fractures, infected fractures, patients with uncontrolled systemic disease or in whom general anesthesia and/or open reduction is contraindicated, mal-union/non-union of fracture segments, and those requiring revision of previous improper treatment. The patients were randomly assigned into two groups Group 1 ( $\mathrm{n}=10$ patients) osteosynthesis by lag screw fixation and Group 2 ( $\mathrm{n}=10$ patients) osteosynthesis by miniplate fixation. All the patients were treated by open reduction and internal fixation under general anesthesia with nasoendotracheal tube on inpatient basis. Surgery for all patients of both groups was don by the same surgical team who was not involved into the research work. Patients prepping and draping was done by a standardized method and the surgical field was prepared by scrubbing with povidone-iodine $1 \%$ antiseptic solution. Access to the fracture site was reached through an intraoral approach by standard surgical incision and dissection for all cases.

After exposure of the fracture line, IMF was applied using arch bars ligated to each of the dental arches and wires between arches to obtain and confirmed an optimum occlusion. The two fractured pieces were reduced into original position with a suitable instrument. The optimum reduction 
confirmed visually and digitally by verifying the all-around alignment of the mandible.

For group 1 two $2.0 \mathrm{~mm}$ diameter and 24-30mm length lag screws were placed according to Ellis guided protocol ${ }^{(11)}, 3 \mathrm{~mm}$ drill used for preparation of gliding hole and $1.5 \mathrm{~mm}$ drill for pilot hole and a specialized tool for countersinking to hidden the screw head then when tighten the screw the two fractured segments were compressed together. The second lag screw was used applied in the same manner. (fig. 1-3). For group 2, two $2.0 \mathrm{~mm}$ miniplates were prepared and adapted on the target area according to the guided protocol using two monocortical 6-9mm length screws on each side of the fracture line according to Champy's principles ${ }^{(7)}$ (fig. 4-6). The time for hard ware preparation and placement were recorded by independent observer for all cases of both groups. After completion of the procedure, the MMF was removed and the occlusion was verified. The wound then irrigated with copious amount of saline and closed in layers. Antibiotics and analgesics were prescribed for all patients for 5 days postoperative.All patients were followed up clinically at the $1^{\text {st }}$ day, $1^{\text {st }}$ week, $1^{\text {st }}$ month, $3^{\text {rd }}$ month and $6^{\text {th }}$ month postoperatively and radiographically by digital panoramic view at the $1^{\text {st }}$ day $1^{\text {st }}$ month, $3^{\text {rd }}$ month and $6^{\text {th }}$ month postoperative.

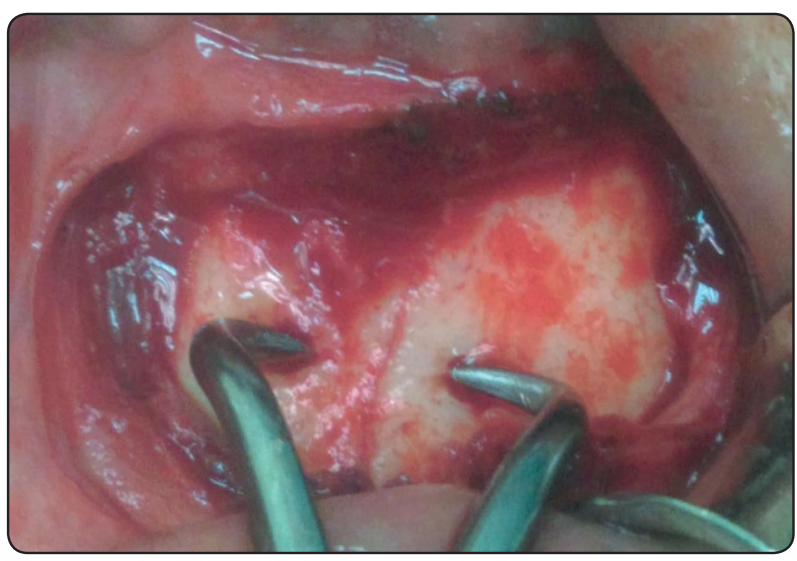

Fig. (1) Fracture line for a case from group 1.
The assessment parameters included the following. The interfragmentary gap which measured preand post-operatively by specific measurement tool in the digital panoramic software, in which a line was drawn along the fracture and divided into three equal distant resulting in four points for measurements, the average value was obtained from these pints represented the final value. ${ }^{(11)}$ Occlusion quality is measured and was considered satisfactory when there was no gap in maximum intercuspation relation and the patient can occlude on both sides perfectly and perform normal function. The occlusion considered mild deranged if there was (1-2mm) gap, where it was considered deranged if there was more than $2 \mathrm{~mm}$ gap. fracture stability was evaluated by manual mobilization of the fracture segments any movement was considered indication for instability. Mastication efficiency was evaluated by recording the ability of the patient to chew hard, medium, soft or semiliquid food. Mouth opening was evaluated by measuring the interincisal distance by digital Vernier. Post-operative complications included pain, edema, mental nerve paresthesia, wound infection and dehiscence, Non-/mal-union, and hardware exposure also evaluated. All parameter was evaluated and recorded on a standardized prosheet by a blinded observer.

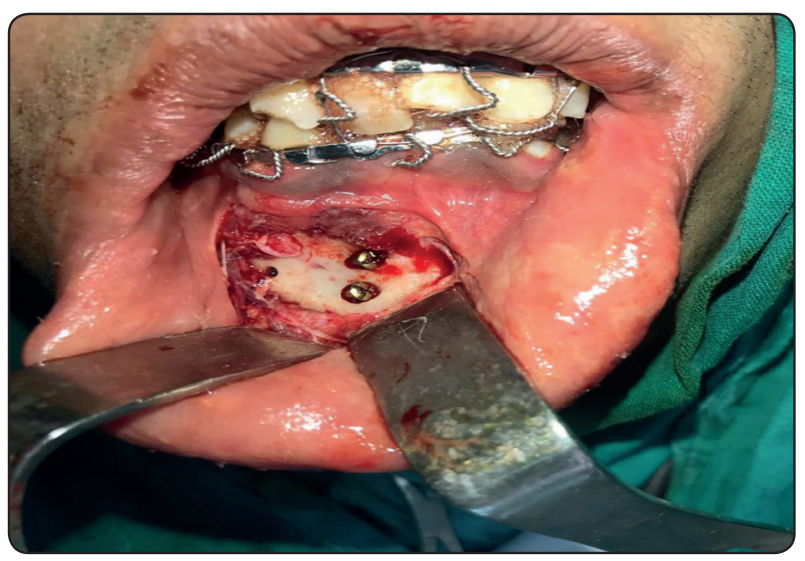

Fig. (2) Lag screw fixation. 


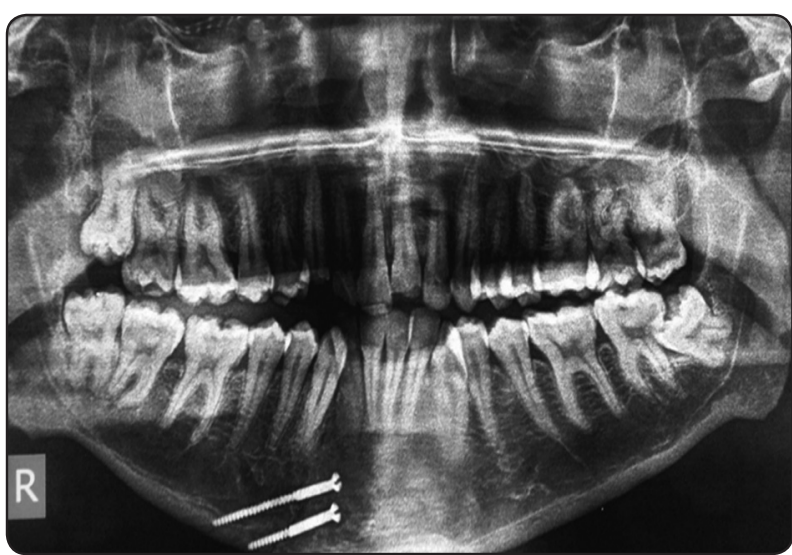

Fig. (3) Panoramic view for lag screw fixation.

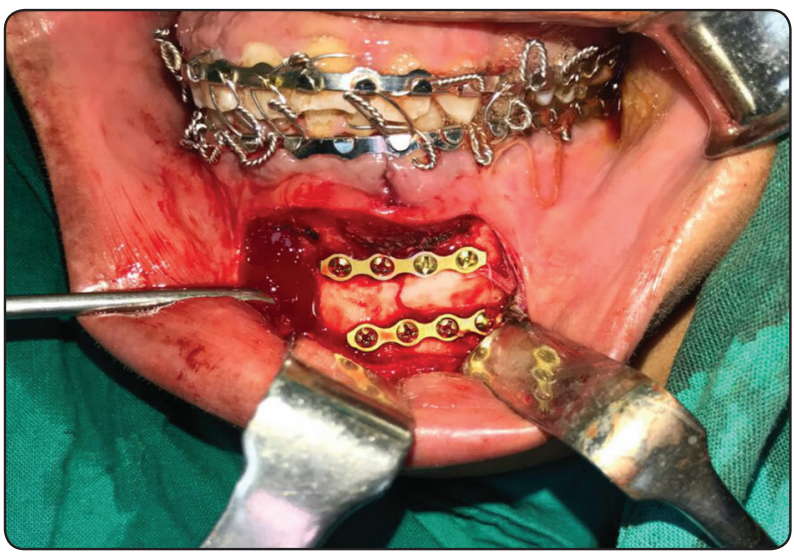

Fig. (5) Miniplates fixation.

\section{RESULTS}

Twenty patients of anterior mandibular fracture were included in current study 17 males (85\%) and 3 females patients comprising (15\%) of the total studied patients. Patients ages ranged between 16-55 years. The average patient age in lag screw was $34.9 \pm 13.19$ years and in miniplates group was $34.8 \pm 13.26$ years with non-significant difference between both groups as revealed by independent samples t-test at 0.05 level $(\mathrm{t}=49.5$; sign. (2-tailed=0.971). Road traffic accident (RTA) was the main cause for the fracture in most of the patients in this study represented by about 16 patients (80\%), however, sport activities, fall, and interpersonal violence were represented by 1 patient each (fig 7). The average lag time in lag screw group

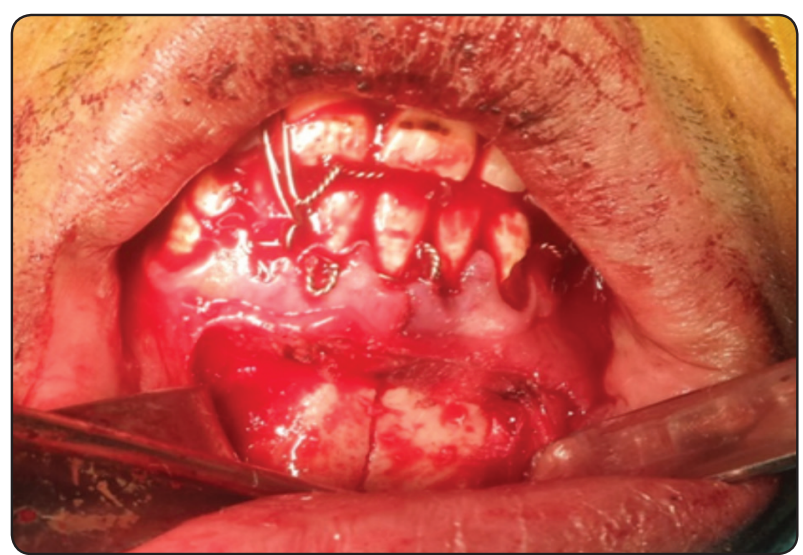

Fig. (4) Fracture line for a case from group 2.

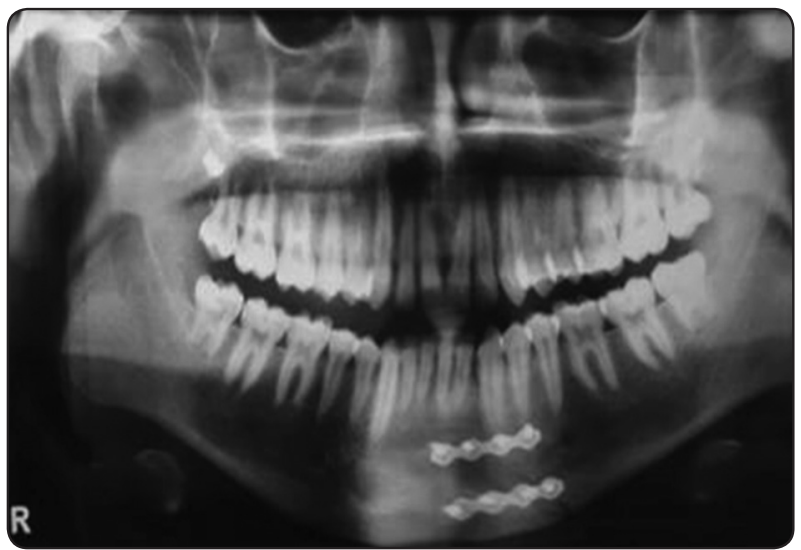

Fig. (6) Panoramic view for miniplates fixation.

was $2.3 \pm 1.33 \mathrm{~d}$, however, in miniplates group was $2.80 \pm 1.55 \mathrm{~d}$ with non-significant difference between both groups as revealed by independent samples $\mathrm{t}$-test at 0.05 level $(\mathrm{t}=-0.77$; sign. $(2$-tailed $)=0.450)$. Hardware fixation time showed a highly significant $(\mathrm{p}<0.001 *)$ difference between group-1 Lag screw with an average of $15.80 \pm 0.80$ minutes and group-2 miniplates with an average of $20.01 \pm 0.823$ minutes. The time for Lag screw fixation (group-1) ranged between 12.10 to 19.80 minutes with an average of $15.80 \pm 0.80$ minutes. The time for miniplates fixation (group-2) ranged between 16.26 to 23.26 minutes with an average of $20.01 \pm 0.82$ minutes. The hardware fixation time of both groups ranged between 12.10 to 23.26 minutes with an overall average of $17.90 \pm 1.04$ (Table 1). 
TABLE (1) Comparative sociodemographic data between group I (Lag screw) and group II (miniplates)

\begin{tabular}{|c|c|c|c|c|c|}
\hline \multirow{2}{*}{ Characteristic } & \multirow{2}{*}{$\frac{\text { Group-I }}{\text { Lag screw }(n=10)}$} & \multirow{2}{*}{$\frac{\text { Group-II }}{\text { Miniplates }(\mathrm{n}=\mathbf{1 0})}$} & \multirow{2}{*}{ Total } & \multicolumn{2}{|c|}{ Significance } \\
\hline & & & & Test statistic & p-value \\
\hline Age in Years $($ Mean + SD) & $34.9 \pm 13.19$ & $34.8 \pm 13.26$ & $34.85 \pm 12.88$ & 49.5 & $0.971 n s$ \\
\hline \multicolumn{6}{|l|}{$\operatorname{Sex}(\%)$} \\
\hline male & $8(80 \%)$ & $9(90 \%)$ & $17(85 \%)$ & \multirow{2}{*}{0.392} & \multirow{2}{*}{$0.500 \mathrm{~ns}$} \\
\hline Female & $2(20 \%)$ & $1(10 \%)$ & $3(15 \%)$ & & \\
\hline \multicolumn{6}{|l|}{ Fracture cause } \\
\hline RTA & $8(80 \%)$ & $8(80 \%)$ & & \multirow{4}{*}{2.00} & \multirow{4}{*}{$0.572 n s$} \\
\hline Sport & $1(10 \%)$ & $0.0(0.0 \%)$ & & & \\
\hline Fall & $1(10 \%)$ & $1(10 \%)$ & & & \\
\hline Fight & $0.0(0.0 \%)$ & $1(10 \%)$ & & & \\
\hline Lag time & $2.3 \pm 1.33$ & $2.80 \pm 1.55$ & $2.55 \pm 1.43$ & -0.77 & $0.450 \mathrm{~ns}$ \\
\hline Operation Time & $15.80 \pm 0.80$ & $20.01 \pm 0.823$ & $17.90 \pm 1.04$ & -3.69 & $0.001 *$ \\
\hline
\end{tabular}

* significant at $p<0.05 ;$ ns non-significant at $p>0.05$

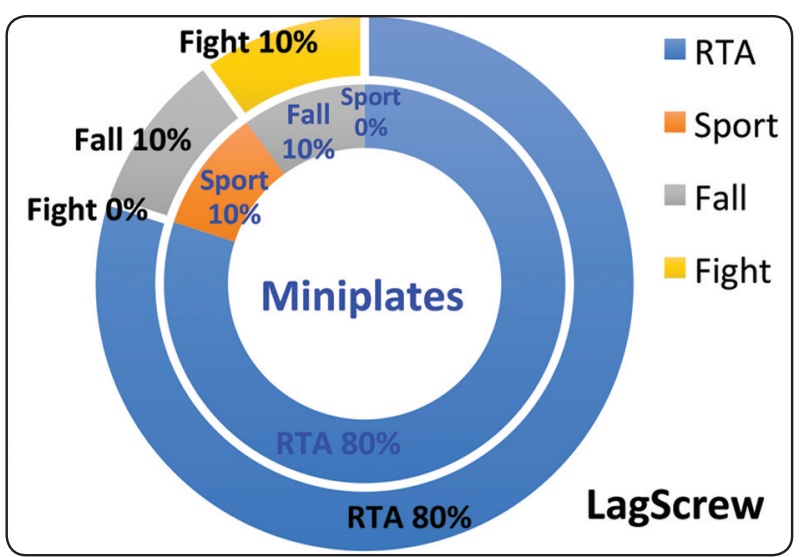

Fig. (7) Doughnut chart representing the fracture cause in both groups.

Interfragmentary distance (gaps) were assessed with the help of the radiographs taken preoperatively and $24 \mathrm{~h}$ postoperatively in both lag screw and miniplates screws (Figure, 3A). The preoperative interfragmentary distances ranged between 0.74 to 4.06 with an average $( \pm \mathrm{SE})$ of $2.42 \pm 1.04$, however, postoperative interfragmentary distance ranged between 0.15 to 1.35 with an average of $0.67 \pm 0.35$. analysis of variance followed by Duncan's multiple range comparisons (DMRTs) revealed that a highly significant decrease in interfragmentary distance from preoperative versus postoperative measures $(\mathrm{p}<0.001 *)$ (Figure 8A). Bars followed by different letters are significantly different according DMRTs at 0.05 level. The difference in interfragmentary distance between lag screw and miniplates treatments were nonsignificant $(\mathrm{p}>0.05)$.

The interincisal mouth opening in in both lag screw and miniplates treatments significantly $\left(\mathrm{p}<0.001^{*}\right)$ increased from preoperative timepoint to postoperative follow-up time $(1 \mathrm{~d}, 1 \mathrm{w}, 1,3$, and 6 months) (figure $8 \mathrm{~B}$ ). The last follow up time point showed non-significant different between lag screw and miniplates treatments as revealed by ANOVA and DMRTs post hoc test at 0.05 level.

Occlusion, Fracture stability, and mastication biting efficiency were improved significantly $\left(\mathrm{p}<0.001^{*}\right)$ in both treatments with time of followup including pre-operative and postoperative (1d, 1w, 1, 3, 6 months) as assessed by Friedman and Cochran's test statistics. Although, a non-significant difference $(p>0.05)$ between both treatments as revealed by Kruskal-Wallis and chi-squared test statistic at 0.05 significance level (Table, 2). 


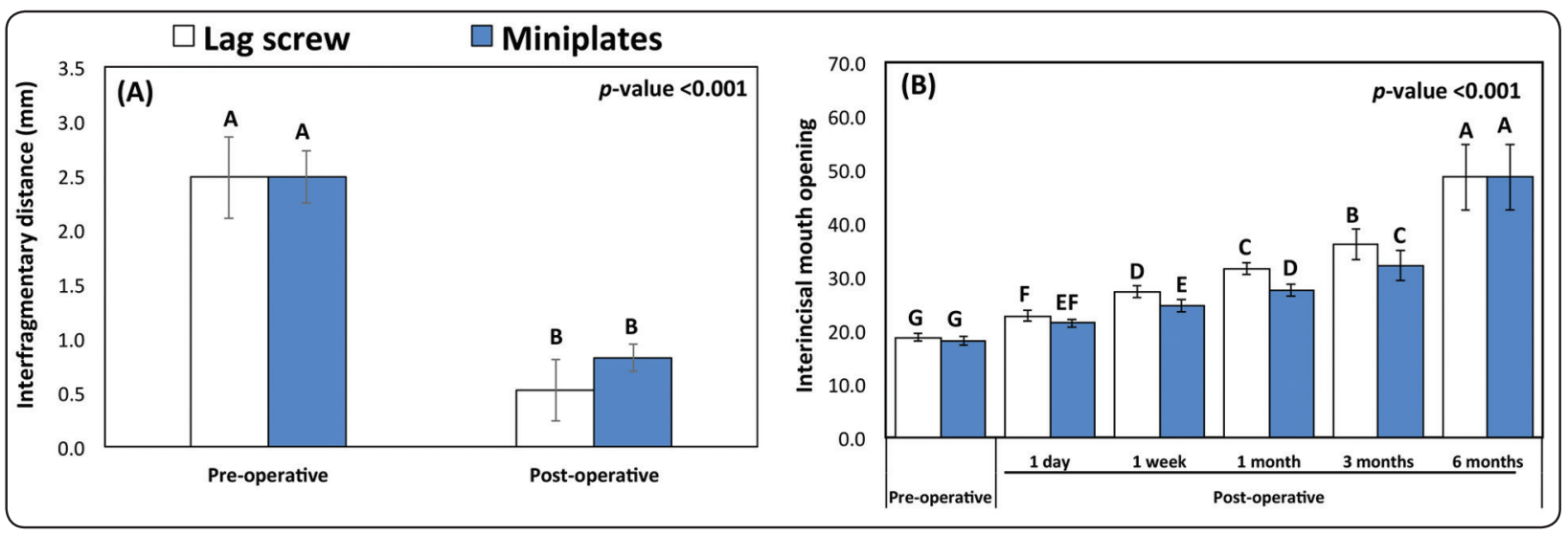

Fig. (8) (A) Interfragmentary distance (mm), (B) interincisal mouth opening (mm) in both groups.

TABLE (2) Occlusion, fracture stability and mastication biting efficiency in both lag screw and miniplates at different follow-up timepoints.

\begin{tabular}{|c|c|c|c|c|c|c|c|c|c|}
\hline \multirow{2}{*}{ Characteristic } & \multirow{2}{*}{ Group } & \multirow{2}{*}{ Scale } & \multirow{2}{*}{$\begin{array}{c}\text { Pre- } \\
\text { operative }\end{array}$} & \multicolumn{5}{|c|}{ Post-operative } & \multirow{2}{*}{$\begin{array}{c}\text { Friedman } \\
\text { Test } \\
\text { Sign. }\end{array}$} \\
\hline & & & & 1 day & 1 week & 1 month & 3 months & 6 months & \\
\hline \multirow{7}{*}{ Occlusion } & \multirow{3}{*}{$\begin{array}{l}\text { Lag screw } \\
\qquad(\mathrm{n}=10)\end{array}$} & Deranged & $10(100 \%)$ & $0(0.0 \%)$ & $0(0.0 \%)$ & $0(0.0 \%)$ & $0(0.0 \%)$ & $0(0.0 \%)$ & \multirow{3}{*}{$<0.001 *$} \\
\hline & & Mild deranged & $0(0.0 \%)$ & $2(20.0 \%)$ & $1(10.0 \%)$ & $0(0.0 \%)$ & $0(0.0 \%)$ & $0(0.0 \%)$ & \\
\hline & & Satisfactory & $0(0.0 \%)$ & $8(80.0 \%)$ & $9(90.0 \%)$ & $10(100 \%)$ & $10(100 \%)$ & $10(100 \%)$ & \\
\hline & \multirow{3}{*}{$\begin{array}{c}\text { Miniplates } \\
\qquad(\mathbf{n = 1 0})\end{array}$} & Deranged & $10(100 \%)$ & $1(10.0 \%)$ & $1(10.0 \%)$ & $2(20.0 \%)$ & $0(0.0 \%)$ & $0(0.0 \%)$ & \multirow{3}{*}{$<0.001 *$} \\
\hline & & Mild deranged & $0(0.0 \%)$ & $3(30.0 \%)$ & $3(30.0 \%)$ & $0(0.0 \%)$ & $1(10.0 \%)$ & $0(0.0 \%)$ & \\
\hline & & Satisfactory & $0(0.0 \%)$ & $6(60.0 \%)$ & $6(60.0 \%)$ & $8(80.0 \%)$ & $9(90.0 \%)$ & $10(100 \%)$ & \\
\hline & $p$-value & & $1.000 \mathrm{~ns}$ & $0.478 \mathrm{~ns}$ & $0.273 \mathrm{~ns}$ & 0.327 ns & $0.500 \mathrm{~ns}$ & $1.000 \mathrm{~ns}$ & \\
\hline \multirow{5}{*}{$\begin{array}{l}\text { Fracture } \\
\text { Stability }\end{array}$} & Lag screw & Unstable & $10(100 \%)$ & $0(0.0 \%)$ & $0(0.0 \%)$ & $0(0.0 \%)$ & $0(0.0 \%)$ & $0(0.0 \%)$ & \multirow{2}{*}{$<0.001 *$} \\
\hline & $(n=10)$ & Stable & $0(0.0 \%)$ & $10(100 \%)$ & $10(100 \%)$ & $10(100 \%)$ & $10(100 \%)$ & $10(100 \%)$ & \\
\hline & \multirow{2}{*}{$\begin{array}{l}\text { Miniplates } \\
\qquad(\mathrm{n}=10)\end{array}$} & Unstable & $10(100 \%)$ & $0(0.0 \%)$ & $0(0.0 \%)$ & $0(0.0 \%)$ & $0(0.0 \%)$ & $0(0.0 \%)$ & \multirow{2}{*}{$<0.001 *$} \\
\hline & & Stable & $0(0.0 \%)$ & $10(100 \%)$ & $10(100 \%)$ & $10(100 \%)$ & $10(100 \%)$ & $10(100 \%)$ & \\
\hline & $p$-value & & $1.000 \mathrm{~ns}$ & $1.000 \mathrm{~ns}$ & $1.000 \mathrm{~ns}$ & $1.000 \mathrm{~ns}$ & $1.000 \mathrm{~ns}$ & $1.000 \mathrm{~ns}$ & \\
\hline \multirow{9}{*}{$\begin{array}{c}\text { Biting } \\
\text { efficiency }\end{array}$} & \multirow{4}{*}{$\begin{array}{l}\text { Lag screw } \\
\qquad(n=10)\end{array}$} & Semi-liquid & $10(100 \%)$ & $0(0.0 \%)$ & $0(0.0 \%)$ & $0(0.0 \%)$ & $0(0.0 \%)$ & $0(0.0 \%)$ & \multirow{4}{*}{$<0.001 *$} \\
\hline & & Soft & $0(0.0 \%)$ & $10(100 \%)$ & $2(20.0 \%)$ & $0(0.0 \%)$ & $0(0.0 \%)$ & $0(0.0 \%)$ & \\
\hline & & Medium & $0(0.0 \%)$ & $0(0.0 \%)$ & $6(60.0 \%)$ & $2(20.0 \%)$ & $0(0.0 \%)$ & $0(0.0 \%)$ & \\
\hline & & Hard & $0(0.0 \%)$ & $0(0.0 \%)$ & $2(20.0 \%)$ & $8(80.0 \%)$ & $10(100 \%)$ & $10(100 \%)$ & \\
\hline & \multirow{4}{*}{$\begin{array}{l}\text { Miniplates } \\
\qquad(\mathrm{n}=10)\end{array}$} & Semi-liquid & $10(100 \%)$ & $0(0.0 \%)$ & $0(0.0 \%)$ & $0(0.0 \%)$ & $0(0.0 \%)$ & $0(0.0 \%)$ & \multirow{4}{*}{$<0.001 *$} \\
\hline & & Soft & $0(0.0 \%)$ & $10(100 \%)$ & $10(100 \%)$ & $5(50.0 \%)$ & $0(0.0 \%)$ & $0(0.0 \%)$ & \\
\hline & & Medium & $0(0.0 \%)$ & $0(0.0 \%)$ & $0(0.0 \%)$ & $5(50.0 \%)$ & $5(50.0 \%)$ & $0(0.0 \%)$ & \\
\hline & & Hard & $0(0.0 \%)$ & $0(0.0 \%)$ & $0(0.0 \%)$ & $0(0.0 \%)$ & $5(50.0 \%)$ & $10(100 \%)$ & \\
\hline & $p$-value & & $1.000 \mathrm{~ns}$ & $1.000 \mathrm{~ns}$ & $0.001 *$ & $<0.001 *$ & $0.016 *$ & $1.000 \mathrm{~ns}$ & \\
\hline
\end{tabular}

* Significant at $p<0.05$; ns nonsignificant at $p>0.05$ 
Evaluation of various post-operative complications in both lag screw and miniplate group were represented in Table (3). Post-operative complications include, pain, edema, paraesthesia, wound dehiscence, wound infections, Non-mal-union, and hardware exposure. Difference in postoperative complications scores between both treatment groups lag screw and miniplate was revealed by Kruskal-Wallis and chi-squared test statistic at 0.05 significance level. A non-significant $(\mathrm{p}<0.05)$ difference between both treatment groups as revealed by nonparametric data analysis including KruskalWallis and chi-squared test at 0.05 level.

TABLE (3) Post-operative complications in both lag screw and miniplate groups at different follow-up time points.

\begin{tabular}{|c|c|c|c|c|c|c|c|c|c|}
\hline \multirow{2}{*}{ Characteristic } & \multirow{2}{*}{ Group } & \multirow{2}{*}{ Scale } & \multirow{2}{*}{$\begin{array}{c}\text { Pre- } \\
\text { operative }\end{array}$} & \multicolumn{5}{|c|}{ Post-operative } & \multirow{2}{*}{$\begin{array}{c}\text { Friedman's } \\
\text { Sign. }\end{array}$} \\
\hline & & & & 1 day & 1 week & 1 month & 3 months & 6 months & \\
\hline \multirow{9}{*}{$\begin{array}{l}\text { (Postoperative } \\
\text { Complications) } \\
\text { pain }\end{array}$} & \multirow{4}{*}{$\begin{array}{l}\text { Lag screw } \\
(n=10)\end{array}$} & No pain & $0(0.0 \%)$ & $0(0.0 \%)$ & $2(20.0 \%)$ & $9(90.0 \%)$ & $10(100 \%)$ & $10(100 \%)$ & \multirow{4}{*}{$<0.001 *$} \\
\hline & & Mild & $0(0.0 \%)$ & $0(0.0 \%)$ & $7(70.0 \%)$ & $1(10.0 \%)$ & $0(0.0 \%)$ & $0(0.0 \%)$ & \\
\hline & & Moderate & $0(0.0 \%)$ & $8(80.0 \%)$ & $1(10.0 \%)$ & $0(0.0 \%)$ & $0(0.0 \%)$ & $0(0.0 \%)$ & \\
\hline & & Severe & $10(100 \%)$ & $2(20.0 \%)$ & $0(0.0 \%)$ & $0(0.0 \%)$ & $0(0.0 \%)$ & $0(0.0 \%)$ & \\
\hline & \multirow{4}{*}{$\begin{array}{l}\text { Miniplates } \\
(n=10)\end{array}$} & No pain & $0(0.0 \%)$ & $0(0.0 \%)$ & $0(0.0 \%)$ & $2(20.0 \%)$ & $10(100 \%)$ & $10(100 \%)$ & \multirow{4}{*}{$<0.001 *$} \\
\hline & & Mild & $0(0.0 \%)$ & $0(0.0 \%)$ & $3(30.0 \%)$ & $8(80.0 \%)$ & $0(0.0 \%)$ & $0(0.0 \%)$ & \\
\hline & & Moderate & $0(0.0 \%)$ & $5(50.0 \%)$ & $7(70.0 \%)$ & $0(0.0 \%)$ & $0(0.0 . \%)$ & $0(0.0 \%)$ & \\
\hline & & Severe & $10(100 \%)$ & $5(50.0 \%)$ & $0(0.0 \%)$ & $0(0.0 \%)$ & $0(0.0 \%)$ & $0(0.0 \%)$ & \\
\hline & $p$-value & & $1.000 \mathrm{~ns}$ & 0.175 ns & $0.006 *$ & $<0.003 *$ & $1.000 \mathrm{~ns}$ & $1.000 \mathrm{~ns}$ & \\
\hline \multirow{5}{*}{$\begin{array}{l}\text { (Postoperative } \\
\text { Complications) } \\
\text { Edema }\end{array}$} & & & & & $8(80.0 \%)$ & $10(100 \%)$ & & $10(100 \%)$ & \multirow{2}{*}{$<0.001 *$} \\
\hline & $(n=10)$ & Present & $10(100 \%)$ & $10(100 \%)$ & $2(20.0 \%)$ & $0(0.0 \%)$ & $0(0.0 \%)$ & $0(0.0 \%)$ & \\
\hline & Miniplates & Absent & $0(0.0 \%)$ & $0(0.0 \%)$ & $3(30.0 \%)$ & $10(100 \%)$ & $10(100 \%)$ & $10(100 \%)$ & \multirow{2}{*}{$<0.001 *$} \\
\hline & $(n=10)$ & Present & $10(100 \%)$ & $10(100 \%)$ & $7(70.0 \%)$ & $0(0.0 \%)$ & $0(0.0 \%)$ & $0(0.0 \%)$ & \\
\hline & $p$-value & & $1.000 \mathrm{~ns}$ & $1.000 \mathrm{~ns}$ & $0.035 *$ & $1.000 \mathrm{~ns}$ & $1.000 \mathrm{~ns}$ & $1.000 \mathrm{~ns}$ & \multirow{3}{*}{$<0.001 *$} \\
\hline \multirow{5}{*}{$\begin{array}{l}\text { (Postoperative } \\
\text { Complications) } \\
\text { Paresthesia }\end{array}$} & Lag screw & & Absent & $7(70.0 \%)$ & $8(80.0 \%)$ & $10(100 \%)$ & $10(100 \%)$ & $10(100 \%)$ & \\
\hline & $(\mathbf{n}=10)$ & & Present & $3(30.0 \%)$ & $2(20.0 \%)$ & $0(0.0 \%)$ & $0(0.0 \%)$ & $0(0.0 \%)$ & \\
\hline & Miniplates & & Absent & $6(60.0 \%)$ & $6(60.0 \%)$ & $8(80.0 \%)$ & $10(100 \%)$ & $10(100 \%)$ & \multirow{2}{*}{$<0.001 *$} \\
\hline & $(n=10)$ & & Present & $4(40.0 \%)$ & $4(40.0 \%)$ & $2(20.0 \%)$ & $0(0.0 \%)$ & $0(0.0 \%)$ & \\
\hline & $p$-value & & & 0.648 ns & 0.314 ns & 0.146 ns & $1.000 \mathrm{~ns}$ & $1.000 \mathrm{~ns}$ & \multirow{3}{*}{$<0.003^{*}$} \\
\hline \multirow{5}{*}{$\begin{array}{l}\text { (Postoperative } \\
\text { Complications) } \\
\text { wound } \\
\text { dehiscence }\end{array}$} & Lag screw & & & $10(100 \%)$ & $8(80.0 \%)$ & $10(100 \%)$ & $10(100 \%)$ & $10(10$ & \\
\hline & $(n=10)$ & & Present & $0(0.0 \%)$ & $2(20.0 \%)$ & $0(0.0 \%)$ & $0(0.0 \%)$ & $0(0.0$ & \\
\hline & Miniplates & & & $10(100 \%)$ & $8(80.0 \%)$ & $10(100 \%)$ & $10(100 \%)$ & $10(100 \%)$ & \multirow{2}{*}{$<0.003^{*}$} \\
\hline & $(n=10)$ & & Present & $0(0.0 \%)$ & $2(20.0 \%)$ & $0(0.0 \%)$ & $0(0.0 \%)$ & $0(0.0 \%)$ & \\
\hline & $p$-value & & & $1.000 \mathrm{~ns}$ & 0.709 ns & $1.000 \mathrm{~ns}$ & $1.000 \mathrm{~ns}$ & $1.000 \mathrm{~ns}$ & \multirow{3}{*}{$>0.05 \mathrm{~ns}$} \\
\hline \multirow{5}{*}{$\begin{array}{l}\text { (Postoperative } \\
\text { Complications) } \\
\text { wound } \\
\text { infection }\end{array}$} & Lag screw & & Absent & $10(100 \%)$ & $10(100 \%)$ & $10(100 \%)$ & $10(100 \%)$ & $10(100 \%)$ & \\
\hline & $(n=10)$ & & Present & $0(0.0 \%)$ & $0(0.0 \%)$ & $0(0.0 \%)$ & $0(0.0 \%)$ & $0(0.0$ & \\
\hline & Miniplates & & Absent & $10(100 \%)$ & $10(100 \%)$ & $10(100 \%)$ & $10(100 \%)$ & $10(100 \%)$ & \multirow{2}{*}{$>0.05 \mathrm{~ns}$} \\
\hline & $(n=10)$ & & & $0(0.0 \%)$ & $0(0.0 \%)$ & $0(0.0 \%)$ & $0(0.0 \%)$ & $0(0.0 \%)$ & \\
\hline & $p$-value & & & $1.000 \mathrm{~ns}$ & $1.000 \mathrm{~ns}$ & $1.000 \mathrm{~ns}$ & $1.000 \mathrm{~ns}$ & $1.000 \mathrm{~ns}$ & \\
\hline \multirow{5}{*}{$\begin{array}{l}\text { (Postoperative } \\
\text { Complications) } \\
\text { Non-/mal- } \\
\text { union }\end{array}$} & Lag screw & & Absent & $10(100 \%)$ & $10(100 \%)$ & $10(100 \%)$ & $10(100 \%)$ & $10(100 \%)$ & \\
\hline & $(\mathrm{n}=10)$ & & Present & $0(0.0 \%)$ & $0(0.0 \%)$ & $0(0.0 \%)$ & $0(0.0 \%)$ & $0(0.0 \%)$ & $.05 \mathrm{~ns}$ \\
\hline & Miniplates & & Absent & $10(100 \%)$ & $10(100 \%)$ & $10(100 \%)$ & $10(100 \%)$ & $10(100 \%)$ & \\
\hline & $(n=10)$ & & & $0(0.0 \%)$ & $0(0.0 \%)$ & $0(0.0 \%)$ & $0(0.0 \%)$ & $0(0.0 \%)$ & $>0.05 \mathrm{~ns}$ \\
\hline & $p$-value & & & $1.000 \mathrm{~ns}$ & $1.000 \mathrm{~ns}$ & $1.000 \mathrm{~ns}$ & $1.000 \mathrm{~ns}$ & $1.000 \mathrm{~ns}$ & \\
\hline stone & Lag screw & & Absent & $10(100 \%)$ & $10(100 \%)$ & $10(100 \%)$ & $10(100 \%)$ & $10(100 \%)$ & \\
\hline & $(\mathrm{n}=10)$ & & Present & $0(0.0 \%)$ & $0(0.0 \%)$ & $0(0.0 \%)$ & $0(0.0 \%)$ & $0(0.0 \%)$ & $>0$ \\
\hline$x^{2}$ & Miniplates & & Absent & $10(100 \%)$ & $10(100 \%)$ & $10(100 \%)$ & $10(100 \%)$ & $10(100 \%)$ & $>0.05 \mathrm{~ns}$ \\
\hline & $(n=10)$ & & Present & $0(0.0 \%)$ & $0(0.0 \%)$ & $0(0.0 \%)$ & $0(0.0 \%)$ & $0(0.0 \%)$ & \\
\hline & $p$-value & & & $1.000 \mathrm{~ns}$ & $1.000 \mathrm{~ns}$ & $1.000 \mathrm{~ns}$ & $1.000 \mathrm{~ns}$ & $1.000 \mathrm{~ns}$ & \\
\hline
\end{tabular}

\footnotetext{
* significant at $p<0.05 ;$ ns nonsignificant at $p>0.05$
} 
Pain intensity was decreased in all cases across the follow up period starting from preoperative to postoperative (1d, 1w, 1, 3, 6 months). A $100 \%$ preoperative sever pain was decreased to $80 \%$ moderate pain after 1 day postoperative and to $90 \%$ and $100 \%$ of patient recorded no pain 1 and 3 months postoperative; respectively (Table 3) in Lag screw group. However, in Miniplates, $100 \%$ preoperative sever pain were decreased to $0 \%$ after 1 week, and $100 \%$ no pain recorded 3 months postoperative. The decrease in pain intensity score across follow up period in both groups was statistically highly significant $\left(\mathrm{p}<0.001^{*}\right)$ (Table 3$)$.

Edema was scaled according to the area of operation and was followed up from preoperative to postoperative (1d, $1 \mathrm{w}, 1$, 3, 6 months). In current study both treatment groups lag screw and miniplate significantly $\left(\mathrm{p}<0.001^{*}\right)$ and completely resolved edema in all the cases (100\% of patients) 1 month postoperative (Table, 3 ) as revealed by Friedman's and Cochran's Q test statistic for related samples.

Wound dehiscence and paresthesia as an important postoperative complication were assessed during the patients follow up postoperatively (1d, $1 \mathrm{w}, 1,3,6$ months). Both treatments significantly improved both paresthesia and wound dehiscence as evaluated statistically by Friedman's and Cochran's Q test statistic for related samples at 0.05 level.

Wound healing went uneventful for all cases in which no infection or wound dehiscence were detected. Wound infection, Non-/mal-union, and/or hardware exposure was non-significantly $(p>0.05)$ changed revealing no complications in all cases during study.

\section{DISCUSSION}

The main goals of fracture management in general is to restore function and original form as early as possible without any comorbidities. In case of fracture mandible management, these goals are extended to include regain of the pre fracture occlusion with enough rigid fixation for better healing, low complications and regain stomatognathic functions as early as possible ${ }^{(13)}$. This study evaluated the abilities of mini plates and lag screws to achieve these goals in anterior mandibular fracture.

In this study the incidence of anterior mandibular fracture was more in men $(85 \%)$ than in women (15\%) which matched with many studies (14-16). This may be clarified by the fact that men are more exposed to trauma causes more than women. The patients age range in this study was $16-55$ years. in current study $80 \%$ of cases caused by road traffic accident which represent the principal cause of anterior mandibular fractures similar to many other studies findings ${ }^{(17)}$ and in contrast with other studies which report assault as the main etiologic factor of anterior mandibular fractures ${ }^{(18)}$.

The duration of surgery was measured from the beginning of hard ware preparation and placement until finished the fixation process. The mean duration was (15.80) in lag screw group whereas (20.01) in miniplates group. Lag screw procedure is relatively faster than miniplate by a statistically significant difference ( $p<0.001)$, this related to obviation of the time for plate bending and adaptation especially in the highly contoured area of the anterior mandible. This matched with the observation of another investigators. ${ }^{(19)}$ The short surgical procedure has many advantages as elucidated by many authors like decreased hospital stay time and low infection complications incidence. ${ }^{(14)}$

Although there is (AO/ASIF) recommendation that fixation by miniplates performed within 12 hours, the the time elapsed between injury and operation for fixation (lag time) for all patient in the current study 1- 6 days and no related disturbances were observed. This is in agreement with another studies ${ }^{(20-22)}$. however other studies reported complications and healing disturbances due to prolonged lag time ${ }^{(23,24)}$

The involved patient's biting efficiency was evaluated, and generally all patients in both groups was inclined to intake soft diet for the first 2 weeks. 
patients of group one showed the ability to chewing solid food between $1^{\text {st }}$ week and 3 months intervals while patients of group two still had difficulty to chewing it, but they were able to intake their regular meals. This is attributed to fracture stability and pain experience. ${ }^{(14,25)}$

In this work Fracture primary stability was achieved in both groups in contrary with another work in which lag screw fixation achieve primary stability than miniplate group and some investigators reported that increasing stability is related to increasing the number of screws. ${ }^{(12)}$ The mouth opening was improved gradually in both groups and non of the patients had prolonged malocclusion. This is attributed to stable intraoperative intramaxillary fixation, excellent adaptation of plates, drilling the holes perpendicular to the fracture lines in cases of using lag screws, and verification of meticulous anatomic reduction of the fracture segments as recommended ${ }^{(26)}$.

In this study radiographic evaluation of the fracture interfragmentary gap was done by measuring the mean distance between fracture fragments. The preoperative gap was $(2.42 \pm 1.40)$ preoperatively which reduced to $(0.67 \pm 0.35)$ postoperatively. The mean postoperative gap in patients managed by lag screw was decreased than in miniplates group $(\mathrm{p}<.05)$. This is due to the compression effect of the lag screw which improve healing by primary intention by permitting direct lamellar ossification inside the gap ${ }^{(27,28)}$. It was reported that fracture gap larger than $2 \mathrm{~mm}$ cause delayed union ${ }^{(29)}$ which not noticed in this study.

With respect to postoperative complications, in this study the incidence of major debilitating complications like infection, prolonged paresthesia malunion/ununion and hard ware exposure was not recorded $(0.00 \%)$ this may be due to using highly technical standard aseptic and atraumatic surgical technique. In contrast minor complications like pain, edema, transient paresthesia was minimal in both groups and rapidly improved and these results is consisted with many researchers ${ }^{(30)}$ and in contrast to another ${ }^{(31)}$. Postoperative pain and edema reported more frequently with the miniplates group than lag screw group this attributed to more prolonged surgical time. Paresthesia was persisted for 1 month in 2 cases of miniplates group, this probably due to the close proximity of the mental nerve to the fracture line.

\section{CONCLUSION}

According to the result of this prospective study it is concluded that, the internal fixation of anterior mandibular fractures with lag screw osteosynthesis is, simple successful achieving rapid fixation so no need for plate adaptation, without any major complications and lowest minor complications. Lag screw offers optimal stability, function, minimum materials, inexpensive, minimally invasive, and lowest inflammatory reactions. Lag screw also providing greater compression to the fracture segments decreasing the interfragmentary gap and achieving primary bone healing. However, the procedure is sensitive, needs mor skill expertise surgeon and it is recommended for further studies with large samples to assess the best method for fixation of the anterior mandibular fracture.

\section{REFERENCES}

1. Miloro M, Ghali GE, Larsen PE, Waite PD (2011) Principles of oral and maxillofacial surgery, 3nd edn. BC Decker Inc Hamilton, London, pp 371-382.

2. Thaller SR, McDonald SW (2005) Facial trauma, 1st edn. Marceld Ekkek Inc, New York, pp 381-414.

3. Ghosh R, Gopalkrishnan K. Facial Fractures. J Craniofac Surg. 2018 Jun;29(4):334-340.

4. Morris C, Bebeau NP, Brockhoff H, Tandon R, Tiwana P. Mandibular fractures: an analysis of the epidemiology and patterns of injury in 4,143 fractures. J Oral Maxillofac Surg. 2015 May;73(5):951-59

5. Pickrell B., Hollier L. Evidence-Based Medicine: Mandible Fractures. Plast Reconstr Surg 2017 Jul;140(1):192-200.

6. Champy M, Loddé JP, Schmitt R et al (1978) Mandibular osteosynthesis by miniature screwed plates via a buccal approach. J Maxilllofac Surg 6:14. 
7. Wusiman P., Taxifulati D., Weidong Li, and Moming A. Three-dimensional versus standard miniplate, lag screws versus miniplates, locking plate versus non-locking miniplates: Management of mandibular fractures, a systematic review and meta-analysis. J Dent Sci. 2019 Mar; 14(1): 66-80.

8. Jadwani S., Bansod S. Lag Screw Fixation of Fracture of the Anterior Mandible: A New Minimal Access Technique J Maxillofac Oral Surg. 2011 Jun; 10(2): 176-180

9. Rao E., Naveen S., Chandra R., Kollabathula K., Srirambhatla M., Gandham S. Principle of Lag-Screw Fixation in Mandibular Trauma J Int Soc Prev Community Dent. 2019 May-Jun; 9(3): 282-289.

10. Kumar V., Mehrotra D.,Mohammad S., Singh R.K., Singh V., Singh G., Gambhir S. Anchor lag screw vs conventional lag screw in mandibular fractures: A series of 30 cases J Oral Biol Craniofac Res. 2013 Jan-Apr; 3(1): 15-19

11. Ellis E. Is Lag Screw Fixation Superior to Plate Fixation to Treat Fractures of the Mandibular Symphysis? J Oral Maxillofac Surg 2012;70 (2):875-82.

12. García Carricondo AR, Quesada Bravo FJ, Espín Gálvez F, Parrón Carreño T, Alarcón Rodriguez R. A comparative study between traditional fixation with miniplates and modified lag screws for the treatment of mandibular fractures. Clin Oral Investig. 2018;22(3):1503-1511.

13. Prein J, Kellman RM. Rigid internal fixation of mandibular fractures - Basics of AO technique. Otolaryngol Clin North Am 1987; 20:441-56.

14. Goyal M, Jhamb A, Chawla S, et al: A comparative evaluation of fixation techniques in anterior mandibular fractures using $2.0 \mathrm{~mm}$ monocortical titanium miniplates versus $2.4 \mathrm{~mm}$ cortical titanium lag screws. J Maxillofac Oral Surg 11:442, 2012

15. Ellis E: Open reduction and internal fixation of combined angle and body/symphysis fractures of the mandible: How much fixation is enough? J Oral Maxillofac Surg 71:726, 2013

16. Guruprasad Y, Shetty J, Prabhakar C, Hemavathy O: Efficacy of 2- mm locking miniplates in the management of mandibular fractures without maxillomandibular fixation. Natl J Maxillofac Surg 2:28, 2011.

17. Johansson B, Krekmanov L, Thomsson M: Miniplate osteosynthesis of infected mandibular fractures. J Craniomaxillofac Surg 16:22, 1988.

18. Dediol E, Cvrljevi c I, Dobranic M, Uglesic V: Comparative study between lag screw and miniplate fixation for straight midline mandibular osteotomy. Int J Oral Maxillofac Surg 43:399, 2014
19. Elhussein M, Sharara A, Ragab H: A comparative study of cortical lag screws and miniplates for internal fixation of mandibular symphyseal region fractures. Alexandria Dent J 42:1, 2017.

20. Lee UK, Rojhani A, Herford AS, Thakker JS (2016) Inmediate versus delayed treatment of mandibular fractures: a stratified analysis of complications. J Oral Maxillofac Surg 74:1186-1196.

21. Webb LS, Makhijani S, Khanna M et al (2009) A comparison of outcomes between inmediate and delayed repair of mandibular fractures. Can J Plast Surg 17:124.

22. Barker DA, Park SS (2011) Is fixation of mandible urgent? Laryngoscope 121:906.

23. Barker DA, Oo KK, Allak A, Park SS (2011) Timing for repair mandible fractures. Laryngoscope 121:1160.

24. Bhatnagar A, Bansal V, Kumar S, Mowar A: Comparative analysis of osteosynthesis of mandibular anterior fractures following open reduction using "stainless steel lag screws and mini plates.” J Maxillofac Oral Surg 12:133, 2013.

25. Tiwana PS, Kushner GM, AlpertB.Lag screw fixation of anterior mandibular fractures: a retrospective analysis of intraoperative and postoperative complications. J Oral Maxillofac Surg 2007;65:1180-5.

26. Mittal G, Aggrawal A, Garg R, Sharma S, Rathi A, Sharma V. A clinical prospective randomized comparative study on ostyeosynthesis of mandibular anterior fractures following open reduction using lag screws and miniplates. Natl J Maxillofac Surg. 2017 Jul-Dec;8(2):110-116.

27. Wang R, Liu Y, Wang JH, Baur DA (2017) Effect of interfragmentary GAP on the mechanical behavior of mandibular angle fracture with three fixation desings. A finite element analysis. J Plast Reconstr Aesthet Surg 70:360-369.

28. Claes L, Eckert-Hubner K, Augat P. The fracture gap size influences the local vascularization and tissue differentiation in callus healing. Langenbecks Arch Surg 2003; 388:316-22.

29. Tiwari M, Meshram V, Lambade P, BDS, Fernandes G. Titanium Lag Screw Versus Miniplate Fixation in the Treatment of Anterior Mandibular Fractures. J Oral Maxillofac Surg 77:1031-1039, 2019.

30. Agnihotri A, Prabhu S, Thomas S, A comparative analysis of the efficacy of cortical screws as lag screws and miniplates for internal fixation of mandibular symphyseal region fractures: a randomized prospective study. Int J Oral Maxillofac Surg43;2014: 22-28. 